\title{
Regolith Mining in Shackleton Crater on the Moon: Propellant, Building Materials and Vital Resources Production for a Long Duration Manned Mission
}

\section{Rabagliati ${ }^{1 *}, M$ Devecchi ${ }^{1}$, A Lovagnini ${ }^{1}$, P Pino ${ }^{1}$ and G Thirion ${ }^{2}$}

${ }^{1}$ Polytechnic University of Turin, Corso Duca degli Abruzzi, Italy

${ }^{2}$ ISAE Supaero, 10 avenue du Colonel Roche, France

\begin{abstract}
The return of humans on the Moon is strongly seen as a fundamental step in the journey to Mars. Lunar exploration has the potential to unlock a new domain of advancements and opportunities that would benefit life on Earth and on other worlds. In this framework, In Situ Resource Utilisation (ISRU) is among the most important objectives to be addressed to enable permanent presence on other celestial bodies.

The baseline for this paper is LUnar Propellant Outpost (LUPO) mission, whose aim is to produce liquid Oxygen and Hydrogen from Lunar South Pole regolith water hydrolysis to refuel spacecraft. The first objective of this work is the resizing of the extraction and production systems to match the establishment of a permanent crew of 20 people. The design of the plants is then analysed to satisfy the water and oxygen demands of crew habitats and manned elements operating on the lunar surface. New ways of utilizing resources are also analysed. Propellant production from regolith has as waste product a huge amount of dehydrated regolith (DR) and thus an evaluation of a DR processing strategy in preparation for applications as building material is performed. Furthermore, molecular hydrogen from water hydrolysis can be, when not used for propulsion purposes, considered as a valuable reducing agent in more advanced processes that can be established in the next future, such as regenerative air revitalization units from $\mathrm{CO} 2$. Finally, an evaluation of the possible volatiles that can be extracted while producing water is performed.

Results show how the overall resizing and improvements implemented increase both production volumes and system efficiency, while simultaneously increasing the autonomy of lunar elements. This, along with the opportunities related to DR exploitation, consistently optimizes a basic architecture of lunar ISRU activities and benefits the related economical aspects.
\end{abstract}

\section{Keywords}

In-situ resource utilisation (ISRU), Propellant, Consumables, Building materials, Volatiles

*Corresponding author: L Rabagliati, Polytechnic University of Turin, Corso Duca degli Abruzzi, 24, 10129 Torino TO; Via Caduti di Cefalonia 23, 15012 Bistagno (AL), Italy

Accepted: February 08, 2021; Published: February 10, 2021

Copyright: (C) 2021 Rabagliati L, et al. This is an open-access article distributed under the terms of the Creative Commons Attribution License, which permits unrestricted use, distribution, and reproduction in any medium, provided the original author and source are credited.

Rabagliati et al. Int J Astronaut Aeronautical Eng 2021, 6:045

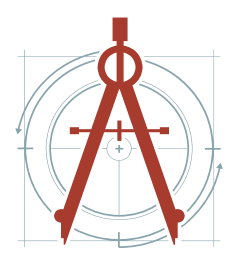




\begin{abstract}
Acronyms/Abbreviations
AHP: Analytic Hierarchy Process; CTV: Crew Transfer Vehicle; DR: Dehydrated Regolith; ECLSS: Environmental Control and Life Support Systems; EVA: Extra-Vehicular Activity; FoM: Figure of Merit; ISRU: In-Situ Resources Utilisation; ISS: International Space Station; LCR: Large Crew Rover; LCROSS: Lunar Crater Observation and Sensing Satellite; LEO: Low Earth Orbit; LH: Liquid Hydrogen; LLO: Low Lunar Orbits; LOX: Liquid Oxygen; LUPO: Lunar Propellant Outpost; NASA: National Aeronautics and Space Administration; NRHO: Near Rectilinear Halo Orbit; PPF: Propellant Production Facility; PSF: Propellant Storage Facility; RAPTOR: Reusable Automated Propellant Transport Rocket; RASSOR: Regolith Advanced Surface Systems Operations Robot; RCS: Regolith Collection System; RTS: Regolith Transportation Systems; SEEDS: Space Exploration and Development Systems
\end{abstract}

\section{Introduction}

According to the words of NASA's Administrator Jim Bridenstine, the next steps of lunar exploration will be performed in such a fashion that "this time, when we go to the Moon, we will stay".

As a result of the Artemis program, NASA hopes to establish a sustained human presence on the Moon by the year 2028 to uncover new scientific discoveries, to demonstrate new technological advancements and lay the foundation for private companies to build a lunar economy [1].

Being the plan to establish a human presence on the Moon and given the high cost of sending materials to the lunar surface, future missions like Artemis will have to learn how to rely on in-situ resources utilisation (ISRU).

In this framework, the Lunar Propellant Outpost (LUPO) is a mission designed by the students of the $2^{\text {nd }}$ level specialising Master SEEDS (Space Exploration and Development Systems) aimed at producing propellant (LH/LOX) on the Moon surface from 2035 until 2050 [2]. Since the starting date of this work was 2018, when the plans of a sustained presence on the Moon surface had not been unveiled yet, the business case of LUPO relied entirely on the propellant market on lunar orbit. Given the new impulse towards a human presence on the Moon, the potential benefits of a surface ISRU market for LUPO's business case have to be analysed.

The identified needs that can be satisfied by an expansion of LUPO infrastructure are propellant for lunar ascenders and consumables and building material for habitats. Each of these three topics will be discussed thoroughly in this paper. No technical aspects such as gravity or temperature related issues will be discussed in the current paper to focus the attention to the high-level possibilities of inter- action between a lunar surface market and LUPO infrastructure. The interested reader in such technical aspects is referenced to $[2,3]$.

\section{The Lunar Propellant Outpost (LUPO)}

The LUPO mission aims to produce propellant on the Lunar surface by exploiting in-situ resources and utilising pre-existing systems, therefore supporting future human space exploration [3]. The propellant selected for the surface production is LH/LOX because of its efficiency when employed in transfer operations between the Lunar surface and Low Lunar Orbits (LLO). Also, it is possible to obtain LH/LOX from the electrolysis of the water present inside the permanently shadowed regions of the craters in the Lunar South Pole. In particular, from the recent analysis of the Chandrayaan-1 M3 data [4], inside the Shackleton crater the regolith is expected to be mixed with ice in quantities from $5 \%$ to $30 \%$ in weight.

The iced-regolith is extracted inside the crater through the Regolith Collection System (RCS) [5] which is based on the NASA's Regolith Advanced Surface Systems Operations Robot (RASSOR) design. The regolith is collected by the Regolith Transportation Systems (RTS) and transported outside the crater to the Propellant Production Facility (PPF). Here, the water is separated from the regolith as water vapour, which is then condensed into liquid water, filtered and finally electrolysed into gaseous Hydrogen and Oxygen [3]. The gases are then cryocooled and stored in the Propellant Storage Facility (PSF).

The mission envisions a Spaceport where the customer's vehicles can dock and refuel. If the customer prefers to be refuelled in LLO, the Reusable Automated Propellant Transport Rocket (RAPTOR) can refuel on the Spaceport and transport the propellant to the refuelling orbit. This would be bene- 
ficial for those missions which have different destinations other than the Lunar surface [6].

At this stage of the preliminary design, there are large uncertainties on both the actual ice percentage inside the extracted regolith and the realistic market's request for propellant on the lunar surface or cislunar space. To accommodate these uncertainties, the ISRU segment is designed relying upon strong modularity. The modular approach is also able to dramatically reduce the cost of the ISRU segment by the advantage of the learning curve, while providing an easier operational set-up and the possibility to increment the business within the mission timeline. In particular, following the preliminary mission timeline [3], LUPO will address the on-orbit market in 2037, two years after the implementation of the first ISRU modules.

Although the capability to address the on-orbit market would largely enhance the propellant request, thus increasing the total revenue, the preliminary business case analysis suggests that on-orbit refuelling could be not so profitable [7]. This is due to the intrinsic limits of the price for the propellant refuelling determined by the cheapest way of reaching a customer's destination. The propellant price is evaluated to be lower for refuelling in LLO (nearly $10 \mathrm{M} \$ / \mathrm{t}$ ) than on the Lunar surface (about $30 \mathrm{M} \$ / \mathrm{t}$ ) [7]. Furthermore, RAPTOR has to consume propellant to reach the refueling orbit and land again on the Spaceport, with an estimated gear ratio of 4 ( $3 \mathrm{~kg}$ of propellant are employed to deliver $1 \mathrm{~kg}$ of propellant in LLO). Thus, from the investor's perspective, the on-orbit market is less attractive because the propellant price has to be lower and $75 \%$ of the propellant produced is wasted in the transport.

From this perspective, the best solution to solve the LUPO business case would be a strong surface market, such as the one related to a permanent Moon Settlement. Such an infrastructure would indeed require a huge amount of propellant to provide transport service of astronauts and goods between lunar orbits (LLO or NRHO) and the surface.

In the following chapter, the potential presence of a human permanent lunar outpost will be taken into account and its relationship with LUPO will be investigated.

\section{A Lunar Surface Market}

As mentioned above, the ambitious goal set by NASA with its Artemis program is to establish a sustained human presence on the Moon by the year 2028. Even if it does not mean to set instantaneously a permanently inhabited human colony on the lunar surface, this will likely happen sooner or later within this century.

As a case study, a lunar base permanently hosting 20 people is assumed to be operative on the lunar South pole within the LUPO time-frame. The analysis of its positive impact on the propellant outpost will be performed, starting from the identification of the basic needs of such settlement which are likely to be satisfied by in-situ resource utilisation.

First of all, the limits imposed by radiation exposure will drive the amount of time that each astronaut will be able to spend in the base. To make some speculations on this topic, 1-year permanence is assumed. This means that an affordable way to bring astronauts back and forth from Earth will have to be present in the overall architecture. The transport system can either bring the propellant from Earth, or it can buy it from LUPO on the Moon surface.

A prolonged human presence in space will also need consumables, in particular water and Oxygen. The amount requested will depend on the technological capability to close the loops of these two resources.

Furthermore, the structures and the radiation shields of permanent habitats will represent a huge percentage of the overall mass of the base. This is why a lot of research on lunar ISRU for building material is currently being performed. There are a lot of possible ways to process regolith to obtain different building resources. A selection of the most promising materials which can benefit from LUPO architecture has to be performed.

Finally, the volatiles extraction and their potential applications will be investigated.

\section{Propellant}

For the sake of the discussion, the transport to the lunar outpost is assumed to be split into two vehicles, one performing the trip from Earth to the Lunar Gateway and back to Earth and the second from the Lunar Gateway to the base and back. Something similar may be studied in case of a direct Earth-Moon transfer with Starship-Super Heavy. 
Focusing on the reusable lander/ascender, to perform a lunar ascent to the lunar gateway and back on the surface would require a total Delta- $V$ of $5500 \mathrm{~m} / \mathrm{s}$ [8]. Assuming a dry mass of $11.4 \mathrm{t}$, as the LUPO Crew Transfer Vehicle (CTV) [2] and a specific impulse of $450 \mathrm{~s}$, a typical value for $\mathrm{LH} / \mathrm{LO}_{2}$ engines, the overall amount of propellant calculated with the Tsiolkovsky equation would be $29 \mathrm{t}$. If the vehicle has a crew capacity of 4 people, the astronauts' shifts will be $5 /$ year and a total propellant request of $145 \mathrm{t} /$ year would be present. The surface market is sized by considering that the cost to ship from Earth to the lunar surface with Starship will be approximately $30 \mathrm{M} \$ / \mathrm{t}$ [7]. This would mean a potential income of about $4 \mathrm{~B} \$ /$ year.

Being the LUPO propellant production facility modular, the change of the demand would not translate into a re-design of the plant and extraction modules: The PPF would only require an additional module. This would increase the efficiency of the mission without affecting its complexity.

The second case would be a Moon-direct with SpaceX Starship-Super Heavy.

Data about Starship-Super Heavy are still unconfirmed and the Delta-V to perform the transfer LEO - Moon surface and back are not easy to predict, because it is probable that aerocapture in LEO will be employed. These two facts impede performing a precise calculation on the amount of propellant that Starship-Super Heavy would require on the Moon surface but having the possibility to refuel on the surface can be attractive from the SpaceX perspective since it can lower the total number of refuelling operations in LEO. Furthermore, the refuelling would consist in Oxygen only, hence the benefit for LUPO would be high because it would not require any change in the original architecture. In fact, LUPO is already producing approximately $250 \mathrm{t}$ of Oxygen more than it can sell, given the selling mixture ratio of LH/LOX engines of $1: 6$ and the production stoichiometric ratio of 1:8.

A critical topic related to this kind of market is the so-called "abort-to-orbit" requirement for crewed vehicles. In fact, if they will have to be capable of aborting the landing at any time of the mission and return to a safe orbit as a safety requirement, no surface refuelling option would be feasible. Nowadays, a definite answer is impossible to obtain, that is why this option is worthy to be evaluated and maintained open until a better understanding of safety regulations will be available.

\section{Consumables}

Designing a manned outpost on the Moon that can host 20 people would be a crucial point for human space exploration. However, by increasing considerably the size of the crew new issues may arise in terms of environmental control and life support. In fact, as compared to the International Space Station (ISS) crew size, a higher quantity of consumables would be required. Currently, the consumables are delivered to the crew by resupply from Earth, but in the near future, thanks to the new capability to exploit the lunar indigenous resources, cheaper solutions may be available.

Astronauts, as living beings, need Oxygen for breathing, potable water and food. Differently from the past missions on the Moon such as the Apollo programme, Oxygen and water needs can be fulfilled with provisions coming from the Moon surface. Considering the scenario with 20 people living permanently on the Moon surface, the daily needs in terms of water and Oxygen are presented in Table 1 [9].

The annual needs are then shown in Table 2. They include the total water and Oxygen required

Table 1: Daily needs per crew member per day [9].

\begin{tabular}{|l|l|l|}
\hline Water needs & Units & Value \\
\hline Drinking-Water & $\mathrm{kg} / \mathrm{CM}$-day & 2.00 \\
\hline Food Rehydration Water & $\mathrm{kg} / \mathrm{CM}$-day & 0.50 \\
\hline Urinal Flush & $\mathrm{kg} / \mathrm{CM}$-day & 0.50 \\
\hline Personal Hygiene & $\mathrm{kg} / \mathrm{CM}$-day & 0.40 \\
\hline Hand wash & $\mathrm{kg} / \mathrm{CM}$-day & 4.10 \\
\hline Shower & $\mathrm{kg} / \mathrm{CM}$-day & 2.70 \\
\hline Medical Water & $\mathrm{kg} / \mathrm{CM}$-day & 0.50 \\
\hline Oxygen needs & $\mathrm{Units}$ & Value \\
\hline $\begin{array}{l}\text { Oxygen consumed in the } \\
\text { habitat }\end{array}$ & $\mathrm{kg} / \mathrm{CM}$-day & 0.83 \\
\hline Leakages in the habitat & $\mathrm{kg} / \mathrm{day}$ & 0.50 \\
\hline EVA Metabolism losses & $\mathrm{kg} / \mathrm{CM}$-day & 0.35 \\
\hline EVA Leakages losses & $\mathrm{kg} / \mathrm{CM}$-day & 0.43 \\
\hline
\end{tabular}

Table 2: Annual needs of 20 people per year.

\begin{tabular}{|l|l|l|}
\hline & Water & Oxygen \\
\hline Need & 102 tonnes & 9.8 tonnes \\
\hline
\end{tabular}


for one year as well as an initial oxygen amount for the pressurisation of the habitat, contingency needs and a safety margin.

In case the habitat cannot recycle water and Oxygen from $\mathrm{CO}_{2}$, the consumables to be supplied would be equal to the needs. If the habitat features a semi-closed loop ECLSS, based on ISS technologies adapted to the Moon environment, the consumables would be much less. In fact, part of the water and the Oxygen consumed by the crew can be recovered by an electrolyser $\left(2 \mathrm{H}_{2} \mathrm{O} \rightarrow 2 \mathrm{H}_{2}+\mathrm{O}_{2}\right)$ and a Sabatier reactor $\left(\mathrm{CO}_{2}+4 \mathrm{H}_{2} \rightarrow 2 \mathrm{H}_{2} \mathrm{O}+\mathrm{CH}_{4}\right)$ (Figure 1).

Different resupply strategies may be applied, according to their types and quantities:

- Only water is supplied to the settlement, which has an increased capability to perform electrolysis to produce Oxygen and Hydrogen. The amount of water to be electrolysed can be sized according to the quantity of Oxygen required by the habitat or to the quantity of Hydrogen employed in the Sabatier reactor. In the first scenario, part of the $\mathrm{CO}_{2}$ produced by the crew has to be vented because of a lack of $\mathrm{H}_{2}$, while in the second scenario a surplus of $\mathrm{O}_{2}$ is produced.

- Oxygen and water are supplied. Without increasing the quantity of water to be electrolysed, only part of the $\mathrm{CO}_{2}$ can be reduced because of the lack of $\mathrm{H}_{2}$. Moreover, less water is produced as a by-product of the Sabatier reaction, thus requiring more water from resupply.

- Hydrogen, Oxygen and water are supplied.

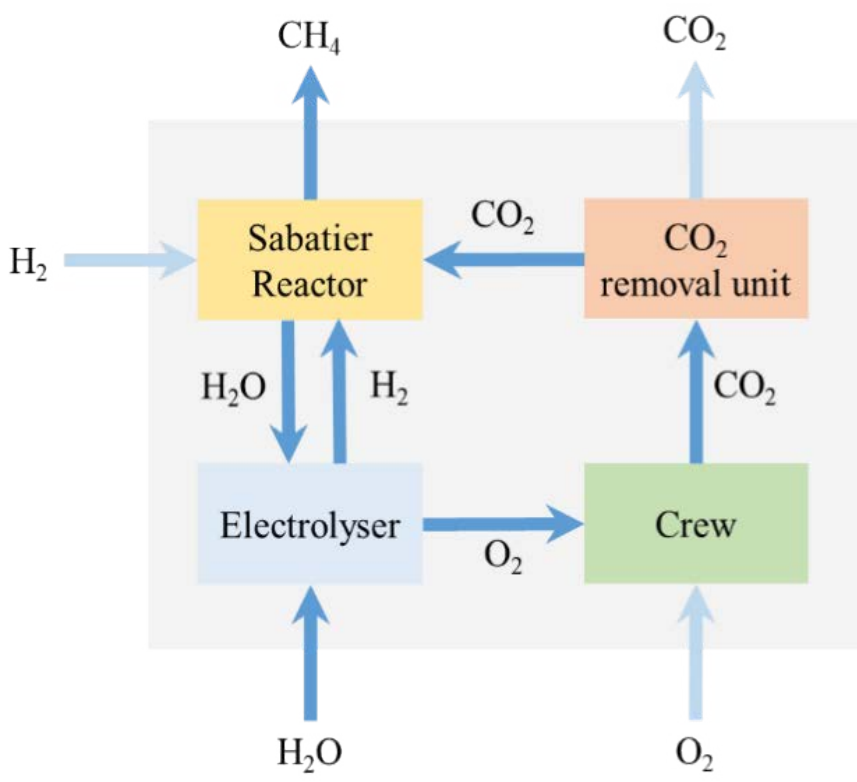

Figure 1: ECLSS schematics: The blurred arrows refer to exchanges that may not occur, according to the scenario considered.

Table 3: Comparison between different ECLSS architectures. All quantities are expressed per year. $\mathrm{R}$ is the percentage of the $\mathrm{CO} 2$ produced by the crew that is recovered by the Sabatier reactor.

\begin{tabular}{|l|l|l|l|l|}
\hline & $\mathbf{H}_{2} \mathbf{O}[\mathbf{t}]$ & $\mathbf{O}_{2}[\mathbf{t}]$ & $\mathbf{H}_{2}[\mathbf{t}]$ & $\mathbf{H}_{2} \mathbf{O}$ electrolysed [t] \\
\hline Open loop & 102 & 9.8 & 0 & 0 \\
\hline Semi-closed loops & & & & \\
\hline $\mathrm{H}_{2} \mathrm{O}(\mathrm{R}=1)$ & 17.8 & 0 & 0 & 13.8 \\
\hline $\mathrm{H}_{2} \mathrm{O}(\mathrm{R}=0.8)$ & 16.8 & 0 & 0 & 11.1 \\
\hline $\mathrm{O}_{2}+\mathrm{H}_{2} \mathrm{O}(\mathrm{R}=0.61)$ & 15.7 & 2.4 & 0 & 8.4 \\
\hline $\mathrm{O}_{2}+\mathrm{H}_{2}+\mathrm{H}_{2} \mathrm{O}(\mathrm{R}=1)$ & 12.4 & 2.4 & 0.6 & 8.4 \\
\hline $\mathrm{O}_{2}+\mathrm{H}_{2}+\mathrm{H}_{2} \mathrm{O}(\mathrm{R}=0.8)$ & 14.1 & 2.4 & 0.3 & 8.4 \\
\hline
\end{tabular}


A first possibility is to recover the entire amount of $\mathrm{CO}_{2}$, thus requiring a high quantity of Hydrogen. An alternative approach could be to partially decrease the $\mathrm{CO}_{2}$ recovered.

The different types of ECLSS are summarised in Table 3.

All these alternatives have advantages and disadvantages and can benefit from in-situ resources. Without performing a proper trade-off, some considerations can be offered, taking into account the relationship with LUPO.

The open-loop architecture is the simplest one and LUPO would enormously benefit from it, given the huge quantity of consumables to provide. However, this solution is less probable to be adopted from a permanent human settlement.

The semi-closed loop scenarios vary in the amount of consumables required, the complexity of the logistics (water is easier to be managed than $\mathrm{O}_{2}$ and $\mathrm{H}_{2}, \mathrm{O}_{2}$ is easier than $\mathrm{H}_{2}$ ) and the complexity of the system that has to electrolyse the water.

From LUPO perspective, the resupply of $\mathrm{O}_{2}+\mathrm{H}_{2} \mathrm{O}$ is the most attractive solution because of the sur- plus of $250 \mathrm{t}$ of oxygen that the propellant facility produces each year. From the settlement point of view, this solution is attractive for the low amount of water to be electrolysed (less mass and power involved) and for the fact that no cryogenic storage of $\mathrm{H}_{2}$ is required. Hence, this alternative is selected.

To enable the water resupply from LUPO, water must be purified before being provided to the crew. According to the LUPO architecture [3], before being electrolysed to get pure Oxygen and Hydrogen, water is purified by the complex ISRU filtration system that consists of multiple ISS-derived filters. In details, the system can manage particulates whose sizes are between $0.5 \mu \mathrm{m}$ and $120 \mu \mathrm{m}$. It is composed of three filters: The particulate filter, the separator filter and the start-up filter [10].

Once the water is purified, it must be mineralized to be used as drinking water. The solution could be to adopt the already proven mineralisation cartridges utilised on the International Space Station.

Subsequently, the logistics of resupply will be studied both for the open-loop and the semi-closed loop case selected. Assuming that the habitat is lo-

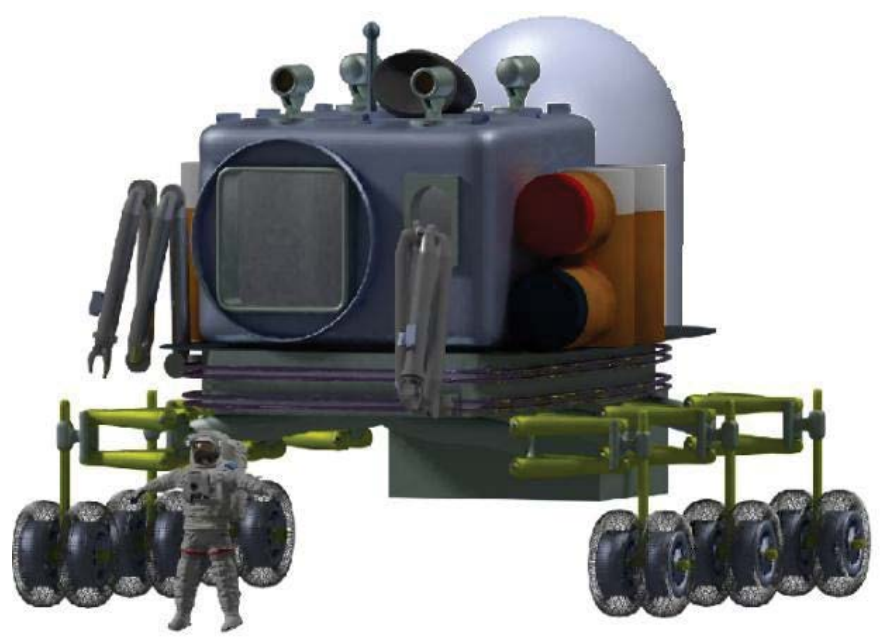

Figure 2: LUPO Large crew rover (LCR).

Table 4: LCR trips per year.

\begin{tabular}{|l|l|l|}
\hline Open-loop & Water supply trips & Oxygen supply trips \\
\hline With pipeline & 0 & 20 \\
\hline With LCR & 204 & 20 \\
\hline Semi-closed loop & Water supply trips & Oxygen supply trips \\
\hline With pipeline & 0 & 5 \\
\hline With LCR & 32 & 5 \\
\hline
\end{tabular}


cated at a distance greater than $1 \mathrm{~km}$ from the PPF due to safety reasons, different alternatives are possible:

- Water is provided through a pipeline connecting the PPF and the habitat. Oxygen is supplied through tanks carried by the Large Crew Rover (Figure 2).

- All the consumables are stored in tanks and carried by the LCR to the habitat.

Considering that the LUPO LCR has a maximum logistic cargo transportation capability of $500 \mathrm{~kg}$ [2], multiple trips would be required for both scenarios to provide all the consumables to the habitat (Table 4).

Each scenario has a different complexity, which takes into account the level of closure of the ECLSS, the number of trips and the need to build a water pipeline. In case the customer does not have any recycling capabilities, a pipeline solution is recommended because of the high number of LCR trips. If the habitat includes a semi-closed loop ECLSS, the provision with the large crew rover (LCR) is suggested.

\section{Building material}

The exploitation of indigenous resources to build habitats and infrastructure is deemed critical to lowering the mass and thus the cost of a lunar permanent mission $[11,12]$. The lunar surface is covered by a layer of unconsolidated debris called regolith. Its composition depends on the location, as shown in Figure 3.

The polar region, where LUPO is located, is best approximated by highlands regolith (to the best of current knowledge) [13]. This means a higher concentration of Aluminium and a lower concentration of Iron and Titanium with respect to mare regions.

The selection of the most suitable material in building applications on the Moon surface is a critical choice since no direct experience with lunar surface structures is available. Nevertheless, a lot of research activities are ongoing, proposing new ways to process regolith for construction applications, resulting in a broad range of alternatives from which to choose when Moon structures will have to be built with indigenous resources.

The most extensive study found in literature about this topic, Happel [14], lists several potential building materials derived from loose regolith and attempts to identify which is the most suitable according to a set of criteria. Starting from that study,

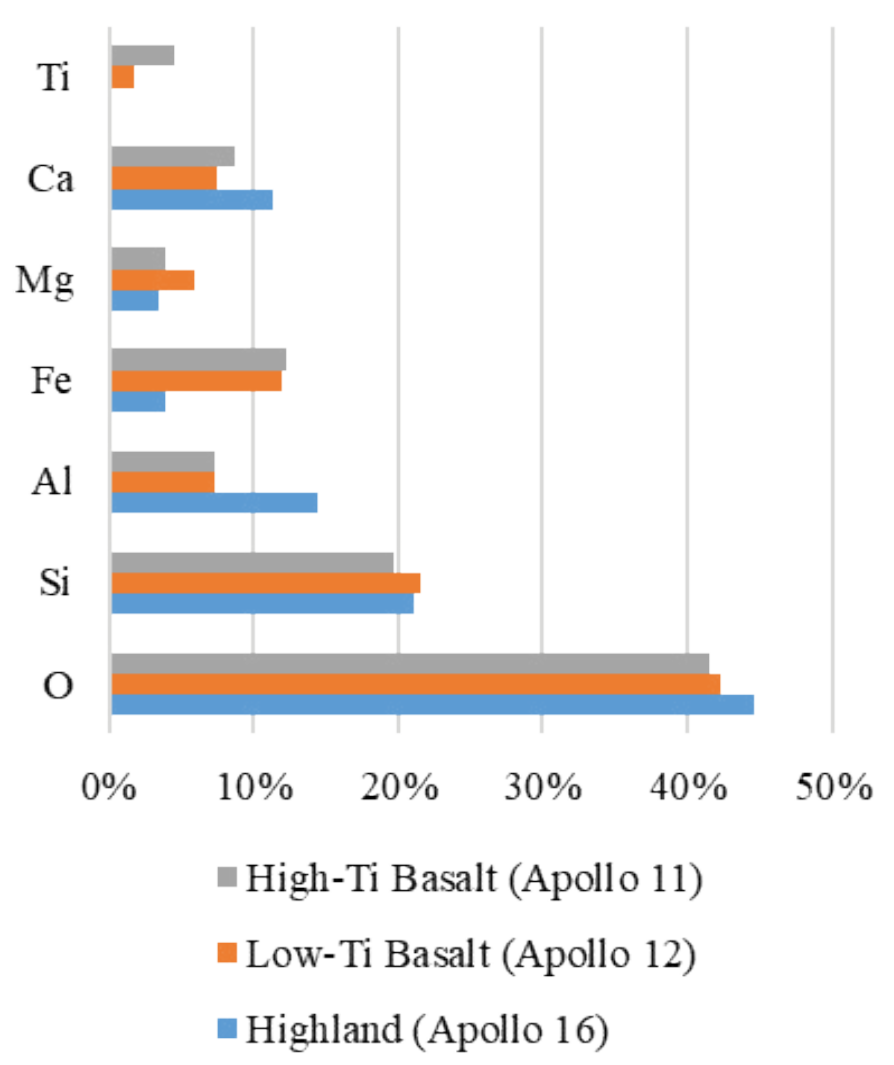

Figure 3: Chemical composition of lunar regolith [12]. 
the trade-off is repeated with three major changes: the scores of the alternatives are updated according to the research of the last decades, the criteria identified to score the alternatives are prioritised with the Analytic Hierarchy Process (AHP) method and a family of criteria, the "Synergy with LUPO", is added.

Alternatives: Different materials are available on the Moon and different ways exist to process them, to eventually manufacture a wide set of products and assets that would, in turn, enable an expanding set of applications and capabilities.

A list of alternative materials has been defined according to the potential ways of exploiting lunar regolith - the most abundant and largely available material on the Moon.

Sintered regolith: Preparing regolith for sintering requires regolith compaction in the desired shape, mixed with a binder where necessary. This process has undergone extensive research over the last years and holds promising benefits in the simple, flexible manufacturing of relatively small parts that can be further assembled, such as bricks [15]. Power demands are generally low and can be sustainably satisfied by concentrated solar energy [16] or by artificial sources such as microwave [17] or laser [18]. Little or no post-processing is required.

Lunar glass: Glass can be easily prepared through simple melting and rapid cooling of lunar regolith. While different mineral compositions might influence the resulting glass properties, nonetheless this should not impact considerably the range of applications, like windows, screens and barriers. Some subsequent thermal treatments would apply. Only little beneficiation would be needed. Vacuum and anhydrous conditions can improve the properties.

Glass composites: A glass production plant could also be integrated with glass fibres production units or other types of ceramic reinforcements that could be embedded in glass matrixed to produce more robust and performing composites. Reinforcements production is however strongly dependent on the desired type and can introduce additional steps and reacting substances, as well as adding complexity to the system.

Cast regolith: Casting regolith can be relatively cheap and easy and can generate large-sized products with different shapes, like slabs, tubes, shields and frames for modular structures [14]. Moreover, almost no beneficiation would be needed, and casting in vacuum would be beneficial. Its brittleness might, however, require the presence of reinforcing parts.

Lunar concrete: As for sintering, many studies have been carried out to assess the potential use of lunar regolith as aggregate for concrete. Different types of cement have been theorized and analysed, either hydraulic [19] or waterless [20]. Lunar concrete would copiously benefit building capabilities and could also take advantage of recently developed automated techniques like contour crafting. On the flipside, preparation might take numerous steps, and cannot be carried in vacuum.

Lunar steel: Iron ores are found in regolith, often in the form of ilmenite $\left(\mathrm{FeTiO}_{3}\right)$, and could be refined via hydrogen or methane reduction among the other processes. Further carbon addition would be necessary to obtain steel and its exceptional benefits. This, along with the relative scarcity of ilmenite in the polar regions, can complicate steel production.

Other metals: Other metals can be derived from lunar regolith employing different extraction processes. Silicon, Aluminium and Titanium are the most interesting ones and could serve in solar cells manufacturing as well as for structural applications or as conductors. Metal extraction processes are usually very energy-intensive and more complex than bare regolith processes, often involving chemical reagents and many steps. The selection of a simple and well-known process is therefore critical in making early lunar metallurgy sustainable.

Criteria: The selection criteria are divided into four families. The first three are related to the process leading to the use of new material in construction [14]: Research and development, extraction and production and operations and utilisation. The fourth added category is the interaction of the process with the LUPO architecture. The list of the sub-criteria will be presented as follows.

Research and development: Knowledge of properties, understanding of utilization, processing simplicity, imported infrastructure.

Production and extraction phase: Raw material availability, process safety, operational ease, automation, maintenance level, environmental hardness, energy request, defect-free production, material testing, incremental expansion, input materials. 


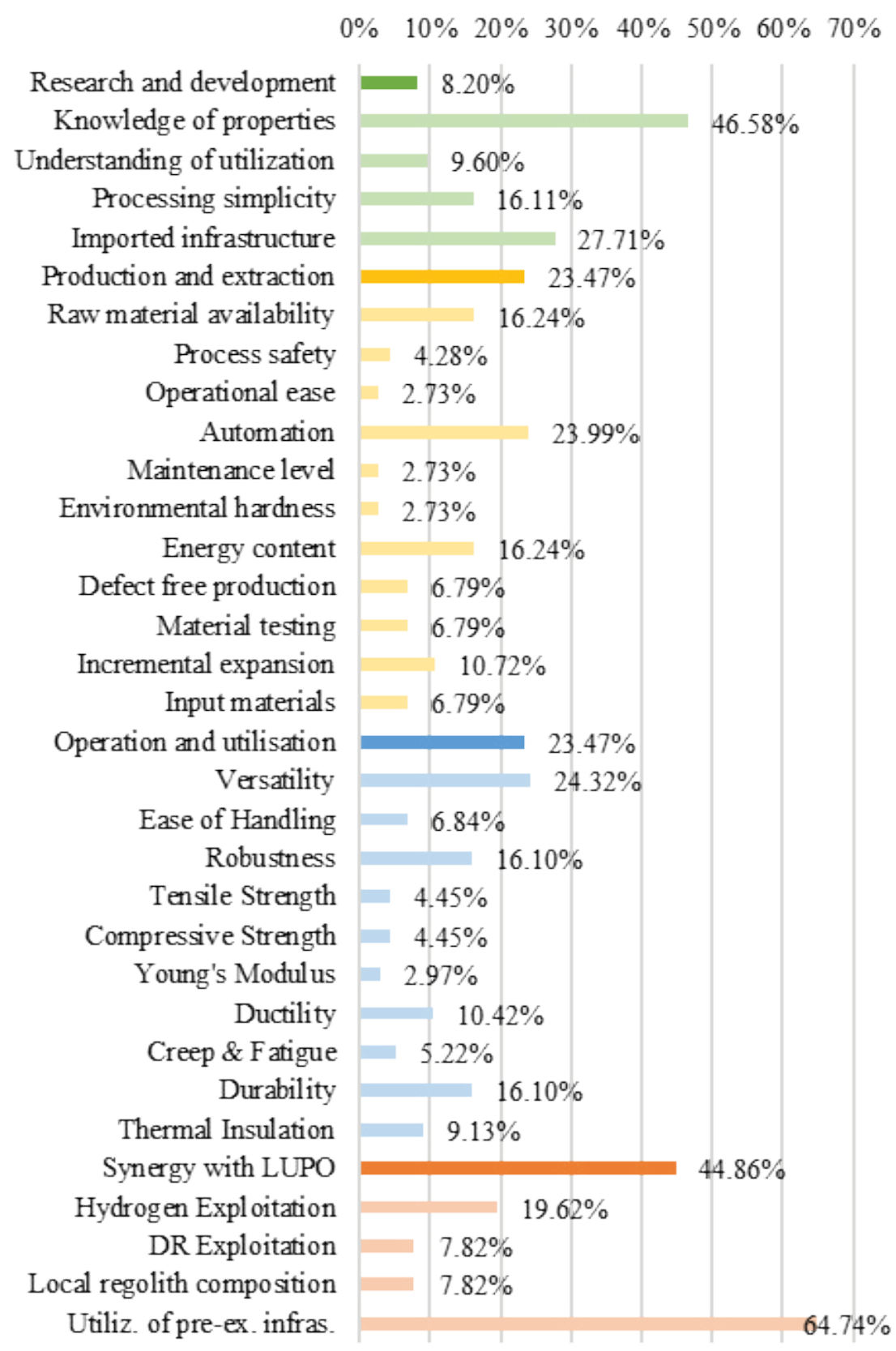

Figure 4: Figures of merit: The blurred bars refers to $2^{\text {nd }}$ level FoMs, whose final weight is evaluated by multiplying its score by the one of its $1^{\text {st }}$ level FoM.

Operations and utilization phase: Versatility, ease of handling, robustness, tensile strength, compressive strength, Young's modulus, ductility, creep \& fatigue, durability, thermal insulation.

Synergy with LUPO: Hydrogen exploitation, dehydrated regolith (DR) exploitation, local regolith composition, Utilisation of pre-existing LUPO infrastructure (PPF, set-up phase).

Results: The AHP method was employed to derive the weights of both the first level and second level criteria (Figure 4): The overall score of each Figure of Merit (FoM) is derived by multiplying its score by the weight of its family.

To each alternative, a score is given for all these criteria. By weighting the score of each alternative with respect to the weight of each criterion, the overall scores are obtained (Figure 5).

Cast regolith was the most appealing construction material identified by Happel [14], followed closely by the lunar steel. The process of updating the scores with data related to the last decades' research did not change this ranking. The most substantial variation is experienced by the lunar concrete, which benefits from the technology ad- 


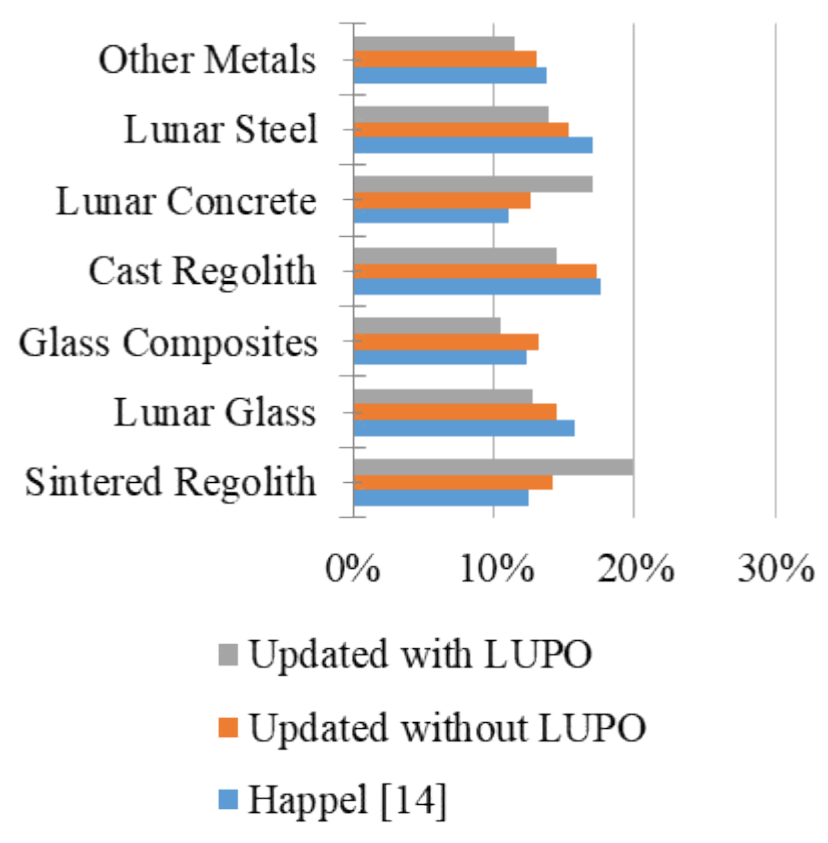

Figure 5: Materials' scores comparison.

vancement in $3 D$ printing and in the better understanding of its properties, due to recent studies focused on it [21,22].

When considering the infrastructure already present within the LUPO architecture, consisting mainly in the utilisation of the LUPO set-up phase $3 \mathrm{D}$ printing rovers, the sintered regolith and the lunar concrete become the most appealing building materials.

The results of the trade-off show that a new revenue stream can be identified in the construction of lunar habitats and infrastructures of a surface lunar base with sintered regolith and/or lunar concrete. Nonetheless, the economic benefits of this potential revenue stream are difficult to be evaluated because of the high number of variables that come into play.

\section{Other resources}

Other resources found on the Moon can increase sustainable ISRU opportunities and valuably impact settlement capabilities and development. Some of them can be successfully derived by the described infrastructure, eventually requesting simple and minor adjustments.

The permanently shadowed regions of the lunar craters are cold traps where volatiles and substances other than water may be found. Analyses of the ejecta plume of the Lunar CRater Observation and Sensing Satellite (LCROSS) impact in the Cabeus cra-
Table 5: Volatiles abundance [25].

\begin{tabular}{|l|l|l|}
\hline Compound & \% Relative to $\mathrm{H}_{2} \mathbf{O}$ & Amount [kg] \\
\hline $\mathrm{H}_{2} \mathrm{O}$ & 100,00 & 1357800 \\
\hline $\mathrm{H}_{2} \mathrm{~S}$ & 16,75 & 227432 \\
\hline $\mathrm{NH}_{3}$ & 6,03 & 81875 \\
\hline $\mathrm{SO}_{2}$ & 3,19 & 43314 \\
\hline $\mathrm{C}_{2} \mathrm{H}_{4}$ & 3,12 & 42363 \\
\hline $\mathrm{CO}_{2}$ & 2,17 & 29464 \\
\hline $\mathrm{CH}_{3} \mathrm{OH}$ & 1,55 & 21046 \\
\hline $\mathrm{CH}_{4}$ & 0,65 & 8826 \\
\hline $\mathrm{OH}$ & 0,03 & 407 \\
\hline
\end{tabular}

ter revealed the presence of chemical compounds in relevant concentrations [23]. Assuming that similar concentrations can be found in the Shackleton crater and knowing the annual production of water from icy regolith according to LUPO architecture [3], the amount of volatiles that can be extracted is estimated in Table 5.

These compounds could be easily separated and collected upon water extraction from regolith at the propellant production facility through fractional distillation [24]. Their applications would be several. The most abundant volatile is Hydrogen Sulphide, which can be employed to recover elemental Sulphur with the Claus process. On Earth, Sulphur is used for manufacturing sulphuric acid, medicine, cosmetics, fertilizers and rubber products, but the 
first application in a future lunar outpost would be being mixed with loose regolith to produce waterless concrete [25]. In this case, the best mechanical properties would be obtained with a sulphur content about $35 \%$ by weight [20], that means that up to $600 \mathrm{t}$ of concrete may be produced each year.

Ammonia and carbon dioxide could be used in greenhouses or as chemical reagents in scientific experiments. Sulphur dioxide can be reduced to obtain again sulphur or oxidised to obtain sulphur trioxide and subsequently reacted with water to obtain sulphuric acid for ilmenite reduction [26]. Carbon-based compounds could be employed for organic chemistry experiments or to produce small quantities of chemical fuels or polymers.

Finally, recycling capabilities shall be seriously considered and eventually implemented on the site. On Earth, aluminium recycling is far more energy-convenient than production via conventional metallurgy. Recycling parts from expendable landers delivered to the surface for set-up or resupply purposes would recover significant amounts of material that could be formed with lower needs for pre-processing or pre-treatment. This would in turn decrease the energy and minerals demands while reducing waste, improving overall the sustainability of the architecture.

\section{Conclusions}

There are many possible opportunities for in-situ resource utilisation, which will be more or less important according to how the Moon will be visited in the future. From the analysis performed in this paper, the propellant production seems very attractive, in particular, if a human presence is established on the Moon surface. In fact, the surface market is much more convenient than the orbit one, since no propellant is wasted to reach the customer in orbit and, at the same time, it is much more valuable there.

Furthermore, a lunar propellant production facility will enable many other possibilities. The consumables demand, water and Oxygen, can easily be satisfied with the same infrastructure the main needs required. Nevertheless, the technology improvements will tend towards close ECLSS loops, which will decrease the amount of consumables required, thus diminishing the importance of an in-situ production. The infrastructure employed for propellant production may be expanded to process the dehydrated regolith, producing lunar concrete and sintered bricks at first, then cast regolith, metals and even glass. Moreover, one of the easiest integrations with the LUPO water extraction is the volatiles extraction, because they are very abundant in the craters regolith with respect to other locations of the Moon surface.

Unfortunately, none of the aforementioned resources is valuable enough to justify its collection and transportation back to Earth. Hence, the role of ISRU will likely be relegated to propellant production until a permanent base on the Moon surface will be present. From that moment on, ISRU will be employed as "import replacement" of goods which are easy to manufacture and/or heavy to transport, such as consumables, structures and radiation shields. Finally, with the evolution of the settlement, more and more needs will drive the collection of resources and volatiles will start to be employed.

As regards the economic viability of these options, especially from a private investor point of view, it is hard to understand whether profitability may be achieved within an acceptable level of risk of the investment. A lot will depend on the governments' plans and their efforts in paving the way to the future lunar economy.

\section{References}

1. (2019) Explore moon to mars. NASA.

2. Guardabasso P, Artuso G, Bigi G, Carré A, Walewski AC, et al. (2018) Lunar outpost sustaining human space exploration by utilizing in-situ resources with a focus on propellant production. IAC-18-A5.1.5, $69^{\text {th }}$ International Astronautical Congress (IAC), Germany, 1-5.

3. Botta S, Adamson I, Barbero D, Beauvois E, Bertolotto $S$, et al. (2019) Lunar propellant factory mission design to sustain future human exploration. IAC19-A5.1.9, $70^{\text {th }}$ International Astronautical Congress (IAC), USA, 21-25.

4. Li S, Lucey PG, Milliken RE, Hayne PO, Fisher E, et al. (2018) Direct evidence of surface exposed water ice in the lunar polar regions. Proceedings of the National Academy of Sciences 115: 8907-8912.

5. Rabagliati L, Karnal M, Pino P, Bertolotto S, Nambiar S, et al. (2019) Thermal challenges related to lunar in-situ resources utilization: Analysis of a regolith mining system. $8^{\text {th }}$ European Conference for Aeronautics and Space Sciences (EUCASS), Spain, Madrid, 1-4. 
6. Cichan T, Bailey SA, Antonelli T, Jolly SD, Chambers RP, et al. (2017) Mars base camp: An architecture for sending humans to mars. In: NEW SPACE 5: 203-218.

7. Pino $P$, Beauvois $E$, Giuliani $M$, Mitchell I, Botta $S$ (2019) Business model for a long duration manned lunar mission: Refuelling, resource commercialisation and new revenue streams. IAC-19-D4.5.5, 70 ${ }^{\text {th }}$ International Astronautical Congress (IAC), USA, 2125.

8. Crusan J (2018) Gateway update, NASA advisory council: Human Exploration and Operations Committee.

9. Anderson MS, Ewert MK, Keener JF, Wagner SA (2018) Life support baseline values and assumptions document. NASA.

10.Jones H (2017) Would current international space station (ISS) Recycling life support systems save mass on a mars transit? $47^{\text {th }}$ International Conference on Environmental Systems.

11.Sacksteder K, Sanders G (2007) In-situ resource utilization for lunar and Mars exploration. $45^{\text {th }}$ AIAA Aerospace Sciences Meeting and Exhibit.

12.Crawford IA (2015) Lunar resources: A review. Progress in Physical Geography 39: 137-167.

13.Schrader CM, Rickman DL, McLemore CA, Fikes JC (2010) Lunar regolith simulant user's guide.

14. Happel JA (1993) Indigenous materials for lunar construction. Applied Mechanics Reviews 46: 313-325.

15.Imhof B, Urbina DA, Weiss $P$, Sperl M, Hoheneder $W$, et al. (2017) Advancing solar sintering for building a base on the moon. $68^{\text {th }}$ International Astronautical Congress (IAC), Adelaide, Australia.
16.Urbina D, Madakashira HK, Salini J, Govindaraj S, Bjoerstad R, et al. (2017) Robotic prototypes for the solar sintering of regolith on the lunar surface developed with in the regolight project. $68^{\text {th }}$ International Astronautical Congress (IAC), Adelaide, Australia.

17. Taylor LA, Meek TT (2005) Microwave sintering of lunar soil: Properties, theory, and practice. Journal of Aerospace Engineering 18: 188-196.

18. Krishna Balla V, Roberson LB, Connor GWO, Trigwell $S$, Bose S, et al. (2012) First demonstration on direct laser fabrication of lunar regolith parts. Rapid Prototyping Journal 18: 451-457.

19. Markandeya Raju P, Pranathi S (2012) Lunarcrete: A review. Proceedings of AARCV 2012 - International Conference on Advances in Architecture and Civil Engineering 2: 886-891.

20.Omar HA (1993) Production of lunar concrete using molten sulfur. NASA.

21. Meurisse A, Makaya A, Willsch C, Sperl M (2018) Solar 3D printing of lunar regolith. Acta Astronautica 152: 800-810.

22.Goulas A, Friel RJ (2016) 3D printing with moondust. Rapid Prototyping Journal 22: 864-870.

23. Colaprete A, Schultz P, Heldmann J, Wooden D, Shirley $M$, et al. (2010) Detection of water in the LCROSS ejecta plume. Science 330: 463-468.

24. Pettit DR (1985) Fractional distillation in a lunar environment. Lunar Bases and Space Activities of the $21^{\text {st }}$ Century.

25.Toutanji HA, Grugel RN (2009) Performance of "waterless concrete". Concrete Solutions: 215.

26.Schwandt C, Hamilton JA, Frayalan DJ, Crawford A (2012) The production of oxygen and metal from lunar regolith. Planetary and Space Science 74: 49-56. 